Article

\title{
Biosynthesis of Putrescine from L-arginine Using Engineered Escherichia coli Whole Cells
}

\author{
Hongjie Hui, Yajun Bai, Tai-Ping Fan, Xiaohui Zheng and Yujie Cai *(D) \\ The Key Laboratory of Industrial Biotechnology, Ministry of Education, School of Biotechnology, \\ Jiangnan University, 1800 Lihu Road, Wuxi 214222, China; rockyhui@foxmail.com (H.H.); \\ baiyj@nwu.edu.cn (Y.B.); tpf1000@cam.ac.uk (T.-P.F.); zhengxh318@nwu.edu.cn (X.Z.) \\ * Correspondence: yjcai@jiangnan.edu.cn; Tel.: +86-18961727911
}

Received: 26 July 2020; Accepted: 17 August 2020; Published: 19 August 2020 updates

\begin{abstract}
Putrescine, a biogenic amine, is a highly valued compound in medicine, industry, and agriculture. In this study, we report a whole-cell biocatalytic method in Escherichia coli for the production of putrescine, using L-arginine as the substrate. L-arginine decarboxylase and agmatine ureohydrolase were co-expressed to produce putrescine from L-arginine. Ten plasmids with different copy numbers and ordering of genes were constructed to balance the expression of the two enzymes, and the best strain was pACYCDuet-speB-speA. The optimal concentration of L-arginine was determined to be $20 \mathrm{mM}$ for this strain. The optimum $\mathrm{pH}$ of the biotransformation was 9.5 , and the optimum temperature was $45^{\circ} \mathrm{C}$; under these conditions, the yield of putrescine was $98 \%$. This whole-cell biocatalytic method appeared to have great potential for the production of putrescine.
\end{abstract}

Keywords: L-arginine; putrescine; Escherichia coli; whole-cell catalysis; co-expression

\section{Introduction}

Putrescine, also known as 1,4-butanediamine, is an aliphatic diamine with a very unpleasant smell and is one of the biogenic amines [1]. In 1889, it was first isolated from Vibrio cholera, and its common name was derived from its occurrence in rotten meat [2]. In both prokaryotic and eukaryotic cells, putrescine is an essential regulator, which affects cell growth, differentiation, proliferation, and various physiological processes $[3,4]$. In mammals, putrescine regulates the intestinal flora and improves the intestinal immune function [4]. Putrescine is also an important metabolite of the human intestinal flora, which is crucial to their life processes [5]. Treating plants with putrescine positively affects their growth, productivity, and stress tolerance [6]. In agriculture, putrescine can enhance salt-tolerance and extend the shelf life of vegetable crops $[7,8]$. In the plastic industry, putrescine is a co-monomer with adipic acid, to prepare the high-quality industrial plastic nylon 46 , which has excellent solvent resistance and mechanical properties [9-11].

The biological synthesis methods of putrescine have been of great research interest in the past decades around the world. Escherichia coli, modified by gene knockout and stronger promoter replacement, achieved a space-time yield of $0.75 \mathrm{~g} \mathrm{~L}^{-1} \mathrm{~h}^{-1}$ with glucose as the substrate, in a high-density culture [10]. Ornithine decarboxylase was successfully modified to achieve a higher catalytic activity, by rational design and molecular docking [12]. Corynebacterium glutamicum was engineered to construct a high-yielding strain (NA6) through gene knockout and metabolic regulation, with L-arginine as the substrate [13-16]. Putrescine has been synthesized in C. glutamicum, using xylose as the substrate [17]. Putrescine was synthesized from agmatine, via the agmatine deiminase pathway by Lactococcus lactis [18]. A combination of various putrescine synthesis pathways in Saccharomyces cerevisiae, with glutamic acid as the substrate, achieved a putrescine concentration of $86 \mathrm{mg} \mathrm{L}^{-1}$ after $48 \mathrm{~h} \mathrm{[19].}$ 
Multienzyme cascade reactions have been widely used to convert cheap substrates into highly valued products. This is a more economical and effective approach than de novo synthesis with glucose under some circumstances [20-22]. In this study, one metabolic pathway for the production of putrescine was constructed using L-arginine as the substrate. In E. coli, L-arginine decarboxylase (ADC) and agmatine ureohydrolase (AUH) were co-expressed. In the first reaction, L-arginine was decarboxylated by ADC to form agmatine and $\mathrm{CO}_{2}$. In the second reaction, $\mathrm{AUH}$ removed urea from agmatine to produce putrescine (Figure 1). E. coli was chosen as the whole-cell catalyst for its excellent protein expression and well-established gene manipulation.

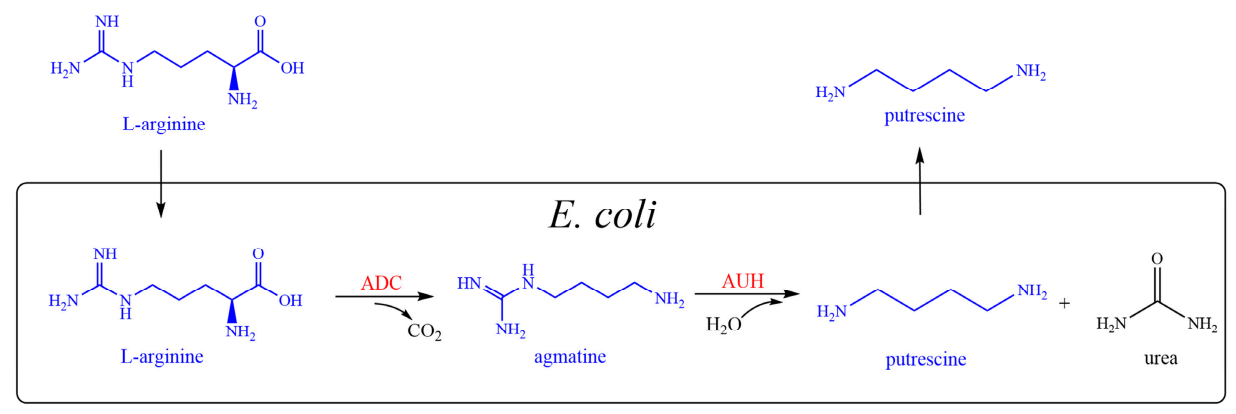

Figure 1. Scheme for the production of putrescine. L-arginine is decarboxylated by ADC to form agmatine and $\mathrm{CO}_{2}$. AUH then removes urea from agmatine to produce putrescine. ADC: $\mathrm{L}$-arginine decarboxylase, encoded by speA; AUH: agmatine ureohydrolyase, encoded by speB.

\section{Results}

\subsection{Construction of E. coli Whole-Cell Biocatalysts}

First, 10 plasmids were constructed using five plasmids with different copy numbers and different orders of spe $A$ and speB. The 10 constructed strains were used as whole-cell biocatalysts to convert L-arginine to putrescine, and each reaction mixture was derivatized with o-phthaldialdehyde and monitored by high performance liquid chromatography (HPLC). A new peak having the same retention time ( $\sim 30 \mathrm{~min})$ as standard putrescine appeared in the reaction mixture, which was confirmed to be the product putrescine (Figure 2). Sodium dodecyl sulfate polyacrylamide gel electrophoresis (SDS-PAGE) showed that the sizes of the two enzymes were approximately $70 \mathrm{kDa}$ and $30 \mathrm{kDa}$, in agreement with the calculated theoretical masses (Figure 3), which were also consistent with previous reports $[23,24]$. Therefore, a biocatalytic synthesis of putrescine from L-arginine as the substrate was successfully engineered.
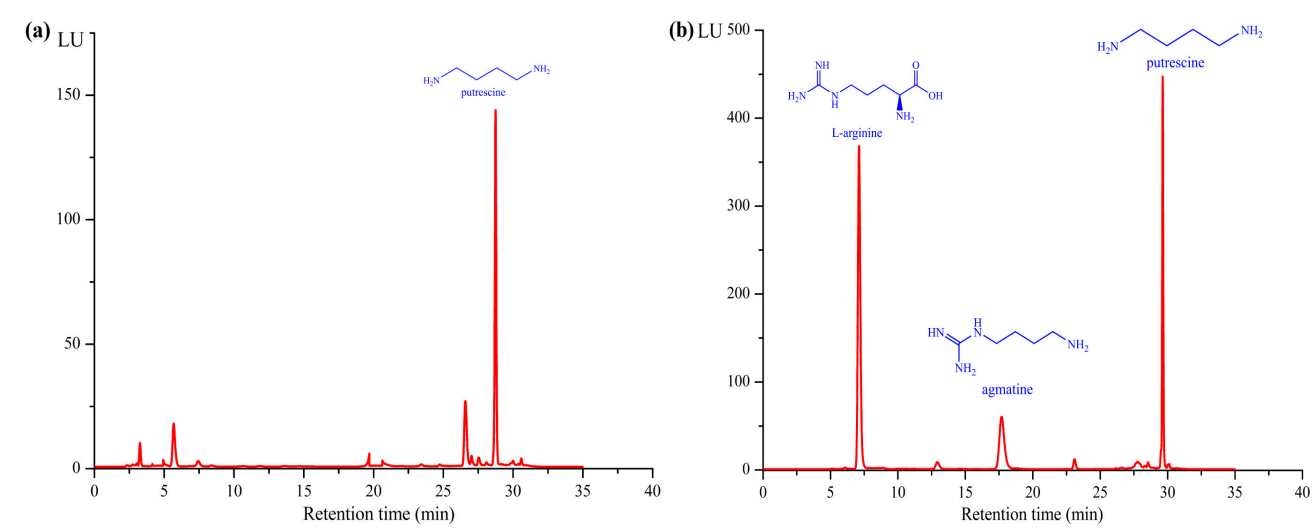

Figure 2. Detection of L-arginine, agmatine, and putrescine. (a) HPLC profile of standard putrescine. (b) The HPLC profile of the product from the catalytic reaction using the whole-cell catalysis. The retention times of L-arginine, agmatine, and putrescine were 7.4, 17.7, and $29 \mathrm{~min}$, respectively. The sample was collected after the reaction for $30 \mathrm{~min}$. 


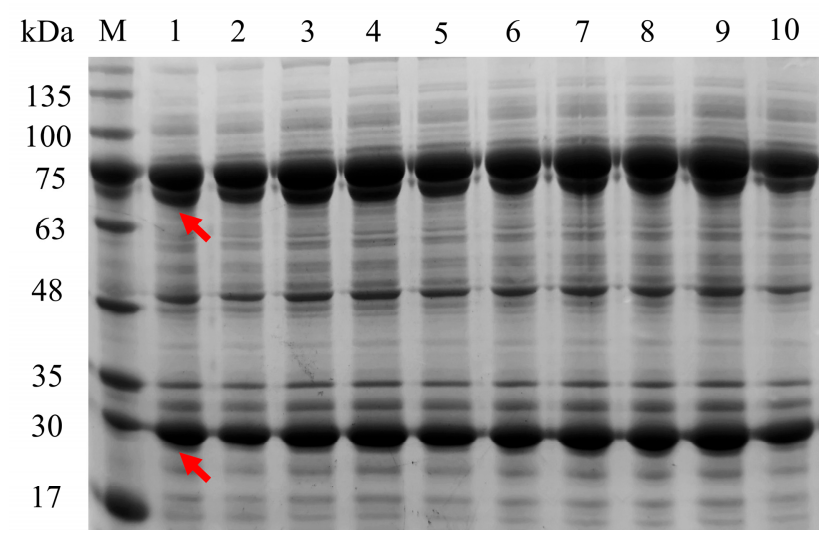

Figure 3. SDS-PAGE (12\% acrylamide) analysis of ADC ( 70 kDa) and AUH ( 30 kDa) from strain 1 to strain 10, which were in agreement with the calculated theoretical masses. Lane M: molecular weight markers. The crude enzymes of strain 1 to strain 10 were shown from lane 1 to lane 10, correspondingly. Marker samples of $5 \mu \mathrm{L}$ and $10 \mu \mathrm{L}$ were loaded, respectively.

\subsection{Comparison of Different Strains for Putrescine Productiond}

The effects of plasmids with different copy numbers and differently ordered genes on the enzymatic process were compared. The differences in the order of the genes markedly influenced the yield of putrescine. The reason may be that the Duet vector contains dual T7 promoters and dual multiple cloning sites, but only one T7 terminator, which may lead to differences in the transcription levels of the two genes [25].

The effect of plasmids with different numbers of copies on yield was determined (Figure 4). Among the low-copy plasmids (strains 1-6), strain 4 had the highest yield. Although the medium-copy plasmid pETDuet-1 could successfully express both ADC and AUH, the overall catalytic effect of strains 7 and 8 was low. Strain 9 and strain 10 had the highest copy number, but their yields were lower than that of strain 4 . The reason may be that the high-copy plasmid combination increases the metabolic pressure and growth burden on the host bacteria $[25,26]$. The biomass of strains with low-copy plasmids was generally greater than those with high-copy plasmids [27]. Strain 4, containing low-copy plasmids, appeared to have the optimal balance between bacterial growth and protein expression to maximize putrescine production. Therefore, it was selected for all subsequent experiments.

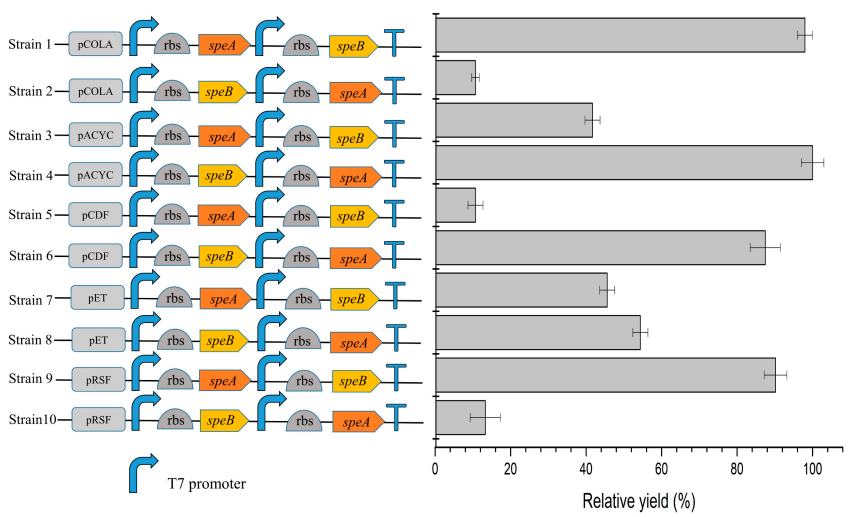

Figure 4. Effects of copy numbers of plasmids and gene orders. The speA and speB encoded ADC and AUH, respectively. The copy number of pCOLADuet-1, pACYCDuet-1, pCDFDuet-1, pETDuet-1, and pRSFDuet- 1 was 5, 10, 20, 40, 100, respectively. Standard deviations are indicated by error bars. 


\subsection{Optimization of Whole-Cell Biotransformation Conditions}

The effect of $\mathrm{pH}$ and temperature on the whole-cell biocatalytic synthesis of putrescine was explored. The optimal biocatalytic temperature of strain 4 was $45^{\circ} \mathrm{C}$ (Figure 5a), and its optimal pH was 9.5 (Figure 5b).


Figure 5. Effects of reaction conditions for strain 4. (a) Effect of temperature. The $\mathrm{pH}$ and L-arginine concentration are 7.5 and $10 \mathrm{mM}$, respectively. (b) Effect of $\mathrm{pH}\left(50 \mathrm{mM} \mathrm{KH}_{2} \mathrm{PO}_{4}-\mathrm{NaOH}\right.$ buffer for $\mathrm{pH}$ 6.5-8.0, 50 mM Tris- $\mathrm{HCl}$ buffer for $\mathrm{pH}$ 8.0-9.0, $50 \mathrm{mM}$ glycine-NaOH buffer for $\mathrm{pH}$ 9.5-10.0, $\mathrm{KCl}-\mathrm{NaOH}$ buffer for $\mathrm{pH} 11.0$ ). The temperature and L-arginine concentration are $45^{\circ} \mathrm{C}$ and $10 \mathrm{mM}$, respectively. (c) Effect of L-arginine concentration. All the experiments were carried out in triplicate. The reaction time is $30 \mathrm{~min}$. Standard deviations are represented by error bars.

The production of putrescine was further studied at different initial concentrations of L-arginine. Under the optimal temperature and $\mathrm{pH}$ conditions, the production of putrescine increased until $20 \mathrm{mM}$ L-arginine was reached, then decreased at higher concentrations (Figure 5c). Higher substrate concentration appeared to produce substrate inhibition. Therefore, $20 \mathrm{mM}$ was selected as the optimal initial L-arginine concentration. In summary, the optimal reaction conditions were: $50 \mathrm{mM}$ Tris- $\mathrm{HCl}$, $20 \mathrm{mM}$ L-arginine, $\mathrm{pH} 9.5,45^{\circ} \mathrm{C}, 1 \mathrm{mM}$ pyridoxal-5'-phosphate, $4 \mathrm{mM}$ magnesium sulfate, and $0.1 \mathrm{mM}$ dithiothreitol. The optimized conditions were used in the next experiment.

\subsection{Time Course of Putrescine Production}

Under the optimal reaction conditions, strain 4 was used to determine the time course of putrescine production over $8 \mathrm{~h}$. The conversion increased to $78 \%$ rapidly in the first $3 \mathrm{~h}$ and then plateaued, presumably because of feedback inhibition by the putrescine product. The conversion only increased by $20 \%$ in the subsequent $5 \mathrm{~h}$. The final yield was $98 \%$ (Figure 6 ).
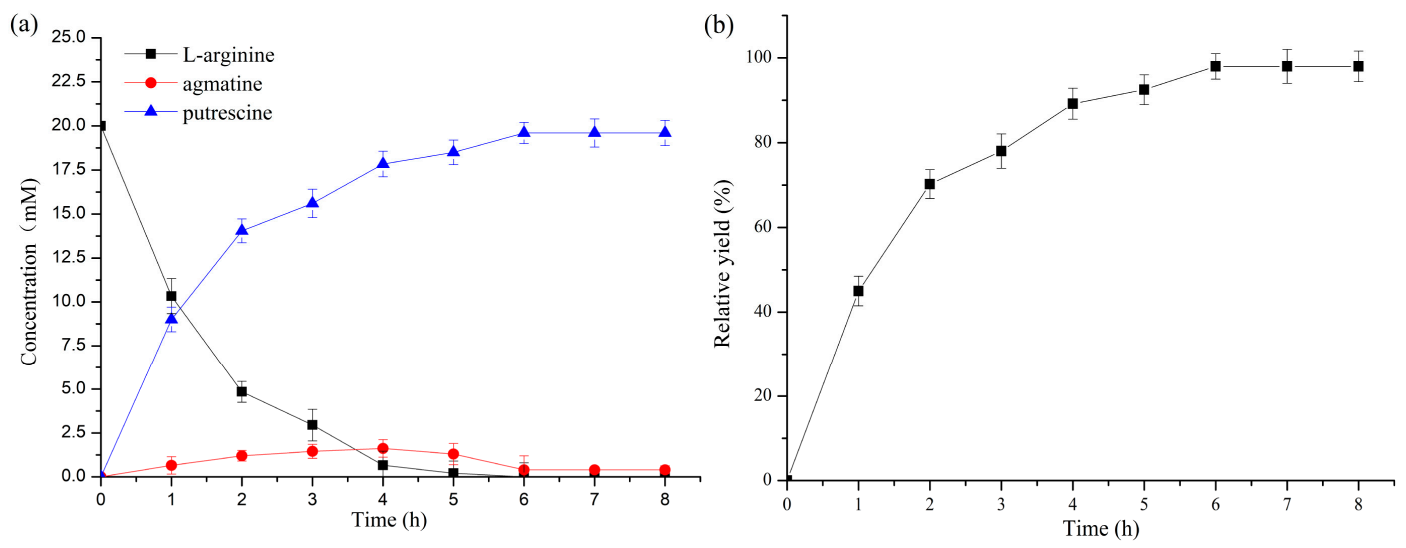

Figure 6. Time course of putrescine synthesis from L-arginine by strain 4. (a) Concentrations of L-arginine, agmatine, and putrescine with time. (b) Mole conversion of putrescine. L-arginine, agmatine, and putrescine were assayed by HPLC. All the experiments were carried out in triplicate. Standard deviations are represented by error bars. 


\section{Discussion}

The biosynthetic pathway for putrescine is widely distributed in microorganisms. There are two biosynthetic pathways for the conversion of L-arginine into putrescine in E. coli. In Pathway I, L-arginine is converted by arginase into ornithine, then, by ornithine decarboxylase into putrescine. If L-arginine is added exogenously, E. coli uses Pathway II, which is the pathway via agmatine, used here (Figure 1) [28]. Both speA and speB are endogenous genes of E. coli and could be expressed effectively.

In the multienzyme cascade reaction system, plasmids with different copy numbers are often used to balance enzyme expression $[25,29,30]$. In this study, five plasmids with different copy numbers were selected to balance the expression of the two enzymes, and thereby optimize the yield of putrescine. Ten engineered strains were constructed based on different combinations. Strain 4 (pACYCDuet-speB-speA) performed the best for the production of putrescine, indicating that low-copy plasmids were better for the production of putrescine than high-copy plasmids. Similarly, in the biosynthesis of N-acetyl-D-neuraminic acid synthesis, the high-copy plasmid pRSFDuet-1 was less efficient than the medium-copy plasmid pCDFDuet-1 [25]. It appeared that, in the selection of plasmid vectors, high-copy plasmids were not always the optimal choice, in some cases, low and medium-copy plasmids performed better in whole-cell biocatalysis.

The optimum temperature of ADC and AUH was $50{ }^{\circ} \mathrm{C}$ and $42{ }^{\circ} \mathrm{C}$, respectively [23,24]. As might be expected, the optimal temperature for whole-cell biocatalysis was $45^{\circ} \mathrm{C}$, between the optima of the two enzymes. The optimum $\mathrm{pH} 9.5$ of the biocatalysis was higher than the individual optima of ADC ( $\mathrm{pH}$ 8.4) and AUH (pH 7.4) [24,31]. This relatively high $\mathrm{pH}$ optimum for the overall process may be related to factors other than the enzymes, such as the cellular uptake of L-arginine, or the intracellular transport of putrescine.

Feedback inhibition is common in cellular biosynthetic pathways, as an essential part of their regulation. $\mathrm{ADC}$ requires $\mathrm{Mg}^{2+}$ as a cofactor and binding of $\mathrm{Mg}^{2+}$ to the enzyme is competitively inhibited by both L-arginine and putrescine [31]. Therefore, $4 \mathrm{mM} \mathrm{Mg}^{2+}$ was added to the reaction buffer to minimize feedback inhibition of cofactor binding. The biocatalytic process had an optimal L-arginine concentration of $20 \mathrm{mM}$, indicating that feedback inhibition occurs at higher concentrations, in agreement with the previous report [31]. Although the increase in $\mathrm{Mg}^{2+}$ concentration reduced the inhibition of putrescine towards ADC, putrescine was still inhibited at high concentrations [31]. There is a complex relationship between the inhibitors and cofactors of ADC, which could be modified by protein engineering, to relieve the feedback inhibition by the biogenic amine [32]. AUH is not inhibited by putrescine, but L-arginine competitively inhibits $\mathrm{AUH}\left(\mathrm{Ki}=9 \times 10^{-3} \mathrm{M}\right)$ [24]. AUH would also benefit from protein engineering, to relieve the inhibitory effect of L-arginine to enhance enzyme activity. The accumulation of the intermediate product agmatine was observed during the cascade reaction (Figure 6a), which does not affect the overall catalytic process. Protein engineering may produce an AUH with higher catalytic activity.

The ornithine decarboxylase (ODC) pathway, with glucose as the substrate, was enhanced in E. coli, achieving a yield $24.2 \mathrm{~g} \mathrm{~L}^{-1}$ after a high-density culture for $32 \mathrm{~h} \mathrm{[10].} \mathrm{In} \mathrm{2012,} \mathrm{the} \mathrm{yield} \mathrm{reached}$ $19 \mathrm{~g} \mathrm{~L}^{-1}$ after high-density fermentation using C. glutamicum for $34 \mathrm{~h} \mathrm{[14].} \mathrm{This} \mathrm{work} \mathrm{started} \mathrm{from}$ L-arginine to produce putrescine by over-expressing two enzymes. After centrifugation, the strains were resuspended with buffer and kept in resting state in the process of catalysis. Other enzymes that may produce side reactions have lower content compared to these two enzymes. The whole-cell biocatalytic process was efficient because of the high permeability of E. coli to the substrate and product, and the high intracellular enzyme concentration. The $0.2 \mathrm{~g} \mathrm{~L}^{-1}$ of dry cell weight in the flask could make the reaction quick and effective, with a short metabolic pathway.

In this study, a whole-cell biocatalytic system was constructed that used L-arginine as the substrate to produce putrescine through dual-enzyme catalysis. L-arginine is a cheap and readily available raw material, which can be converted relatively easily into high-value putrescine [33]. This biosynthetic method has the advantages of simple operation, low cost, and high yield. In the future, industrial production of putrescine could be improved through the in-situ separation of products or molecular 
evolution. Compared with traditional separation methods, in-situ separation methods can reduce the feedback inhibition of products and the use of organic reagents, which is of great significance for the industrial production of putrescine. Based on the characteristics of putrescine, materials like resin could be designed to separate putrescine from the culture broth to improve the yield and fermentation efficiency. The methods of in-situ separation include adsorption resin, ion exchange resin, foam chromatography, ultrafiltration, and so on. At present, it is meaningful and challenging to establish the method of in-situ separation of putrescine, and match well with $E$. coli whole-cell catalysis [34].

\section{Materials and Methods}

\subsection{Strains, Plasmids, Other Materials}

E. coli JM109 and E. coli BL21 (DE3) were used as the cloning host and the expression host, respectively. The plasmids pACYCDuet-1, pCOLADuet-1, pCDFDuet-1, pETDuet-1, and pRSFDuet-1 were from Novagen (Darmstadt, Germany). The DNA gel extraction kit, plasmid miniprep kit, and Taq DNA polymerases were from TaKaRa. The multiF seamless connection kits were from Abclonal (Wuhan, China). The isopropyl $\beta$-D-1-thiogalactopyranoside, ampicillin, kanamycin, chloramphenicol, and streptomycin were from Sangon Biotech (Shanghai, China). Standards: the L-arginine, agmatine sulfate, putrescine, and pyridoxal-5'-phosphate were from Aladdin (Shanghai, China). The methanol and tetrahydrofuran were chromatography grade, from Tedia (Fairfield, OH 45014, U.S.A.) and Rhawn (Shanghai, China), respectively. Primer synthesis and gene sequencing were performed by Talen-bio Scientific (Shanghai, China). The primers used are listed in Table 1.

Table 1. Primers used in the study.

\begin{tabular}{cl}
\hline Primers & \multicolumn{1}{c}{ Sequences, $\mathbf{5}^{\prime} \mathbf{- 3}^{\prime \prime}$} \\
\hline Site1-speA-F & TTAAGTATAAGAAGGAGATATACATATGAGCACCTTAGGTCATCAATACG \\
Site1-speA-R & TTAAGCATTATGCGGCCGCAAGCTTTTACTCATCTTCAAGATAAGTATAAC \\
Site1-speB-F & TCACCACAGCCAGGATCCGAATTCGATGAGCACCTTAGGTCATCAATACG \\
Site1-speB-R & TTTCTTTACCAGACTCGAGGGTACCTTACTCGCCCTTTTTCGCCG \\
Site2-speA-F & TTAAGTATAAGAAGGAGATATACATATGTCTGACGACATGTCTATGGGT \\
Site2-speA-R & TTAAGCATTATGCGGCCGCAAGCTTTTACTCGCCCTTTTTCGCCGC \\
Site2-speB-F & TTAAGCATTATGCGGCCGCAAGCTTTTACTCATCTTCAAGATAAGTATAAC \\
Site2-speB-R & TTTCTTTACCAGACTCGAGGGTACCTTACTCATCTTCAAGATAAGTATAACC \\
\hline
\end{tabular}

\subsection{Pathway and Plasmid Construction}

Using E. coli BL21 (DE3) as the template, speA (GenBank ID: CP032667.1) and speB (GenBank ID: CP028306.1) were amplified by PCR, encoding ADC and AUH, respectively. Plasmids with different copy numbers were selected for comparison: pCOLADuet-1 (low-copy, copy number 5), pACYCDuet-1 (low-copy, copy number 10), pCDFDuet-1 (low-copy, copy number 20), pETDuet-1 (medium-copy, copy number 40), and pRSFDuet-1 (high-copy, copy number 100) [21]. The genes were ligated to the plasmids with multiF seamless connection kits. For each plasmid, two versions with speA and speB in different orders were constructed. The plasmid was transformed into E. coli BL21 (DE3), and the success of the transformation was confirmed by both the presence of the antibiotic resistance gene and by gene sequencing. The plasmids and strains used in this study are listed in Table 2. 
Table 2. Strains and plasmids used in this study.

\begin{tabular}{|c|c|c|}
\hline Strains/Plasmids & Description & Source \\
\hline \multicolumn{3}{|l|}{ Plasmids } \\
\hline pCOLADuet-1 & double T7 promoters, COLA ori, KanR & Novagen \\
\hline pACYCDuet-1 & double T7 promoters, P15A ori, ChlR & Novagen \\
\hline pCDFDuet-1 & double T7 promoters, CDF13 ori, SmR & Novagen \\
\hline pETDuet-1 & double T7 promoters, pBR322 ori, AmpR & Novagen \\
\hline pRSFDuet-1 & double T7 promoters, RSF ori, KanR & Novagen \\
\hline pCOLADuet-speA-speB & pCOLADuet- 1 carrying spe $A$ and speB & this study \\
\hline pCOLADuet-speB-speA & pCOLADuet- 1 carrying speB and spe $A$ & this study \\
\hline pACYCDuet-speA-speB & pACYCDuet- 1 carrying spe $A$ and speB & this study \\
\hline pACYCDuet-speB-speA & pACYCDuet- 1 carrying speB and spe $A$ & this study \\
\hline pCDFDuet-speA-speB & pCDFDuet- 1 carrying spe $A$ and spe $B$ & this study \\
\hline pCDFDuet-speB-speA & pCDFDuet- 1 carrying speB and speA & this study \\
\hline pETDuet-spe $A$-speB & pETDuet- 1 carrying spe $A$ and speB & this study \\
\hline pETDuet-speB-speA & pETDuet- 1 carrying speB and spe $A$ & this study \\
\hline pRSFDuet-speA-speB & pRSFDuet- 1 carrying spe $A$ and speB & this study \\
\hline pRSFDuet-speB-speA & pRSFDuet- 1 carrying speB and spe $A$ & this study \\
\hline \multicolumn{3}{|l|}{ Strains } \\
\hline strain 1 & E. coli BL21 (DE3)/pACYCDuet-speA-speB & this study \\
\hline strain 2 & E. coli BL21 (DE3)/pACYCDuet-speB-speA & this study \\
\hline strain 3 & E. coli BL21 (DE3)/pCOlADuet- speA-speB & this study \\
\hline strain 4 & E. coli BL21 (DE3)/pCOlADuet- speB-speA & this study \\
\hline strain 5 & E. coli BL21 (DE3)/pETDuet- speA-speB & this study \\
\hline strain 6 & E. coli BL21 (DE3)/pETDuet- speB-speA & this study \\
\hline strain 7 & E. coli BL21 (DE3)/pCDFDuet- speA-speB & this study \\
\hline strain 8 & E. coli BL21 (DE3)/pCDFDuet- speB-speA & this study \\
\hline strain 9 & E. coli BL21 (DE3)/pRSFDuet- speA-speB & this study \\
\hline strain 10 & E. coli BL21 (DE3)/pRSFDuet- speB-speA & this study \\
\hline
\end{tabular}

\subsection{Culture Conditions and Preparation of Whole-Cell Biocatalysts}

The seed solution was activated overnight in test tubes containing Luria-Bertani (LB) medium $(3 \mathrm{~mL})$, then added to $50 \mathrm{~mL} \mathrm{LB}$ medium at a ratio of 1:50 (v/v). Various antibiotics were added at the start of the culture, including ampicillin $\left(100 \mathrm{mg} \mathrm{mL}^{-1)}\right.$, kanamycin $\left(40 \mathrm{mg} \mathrm{mL}^{-1}\right)$, chloramphenicol (20 mg mL $\left.{ }^{-1}\right)$, and streptomycin $\left(40 \mathrm{mg} \mathrm{mL}^{-1}\right)$. The strains were incubated for $2 \mathrm{~h}$ in $250 \mathrm{~mL}$ shake flasks at $37^{\circ} \mathrm{C}$ and $200 \mathrm{rpm}$. When the optical density reached 0.6 at $600 \mathrm{~nm}$, isopropyl $\beta$-D-1-thiogalactopyranoside was added to a final concentration of $0.4 \mathrm{mM}$. Subsequently, strains were incubated, in shake flasks for $24 \mathrm{~h}$ at $200 \mathrm{rpm}$ and $15^{\circ} \mathrm{C}$, and the cells were then collected by centrifugation (8000 $\left.\times g, 4{ }^{\circ} \mathrm{C}, 10 \mathrm{~min}\right)$. Finally, they were resuspended in the buffer. E. coli BL21 (DE3), but without the target genes, was used as the control.

\subsection{SDS-PAGE Analysis}

The cells were disrupted with an ultrasonic probe for $20 \mathrm{~min}$ and centrifuged at $5000 \times g$ for $10 \mathrm{~min}$. Then, the denaturing buffer $(10 \mu \mathrm{L})$ was added to the supernatant $(40 \mu \mathrm{L})$ and heated for $10 \mathrm{~min}$ at $100{ }^{\circ} \mathrm{C}$. SDS-PAGE ( $12 \%$ acrylamide) was used for electrophoresis. After electrophoresis, the protein bands were stained with Coomassie Brilliant Blue R250. The bands were imaged using BandScan software version 4.3 , after destaining.

\subsection{Whole-Cell Biocatalysts and Optimization of Reaction Conditions}

The reaction was carried out in a buffer and included the following components: $20 \mathrm{mM}$ L-arginine, $4 \mathrm{mM}$ magnesium sulfate, $1 \mathrm{mM}$ pyridoxal-5'-phosphate, and $0.1 \mathrm{mM}$ dithiothreitol. The reaction was carried out in a $250 \mathrm{~mL}$ shake flask, at $37^{\circ} \mathrm{C}$ and $200 \mathrm{rpm}$. The buffer was used to adjust the cell density of each strain to the same level. Then, the same amount of each strain was used for the 
whole-cell catalysis trials. To start the reaction, bacterial cells (10 $\mathrm{mg}$ dry weight) were added to the reaction solution $(50 \mathrm{~mL})$ for $30 \mathrm{~min}$. Finally, 1/5 volume of trichloroacetic acid was added to terminate the reaction.

To evaluate the effect of temperature on biotransformation, the reaction was carried out at 20, 30, 40,50 , and $60^{\circ} \mathrm{C}$. In order to evaluate the influence of $\mathrm{pH}$, the $\mathrm{pH}$ of the reaction system was adjusted to $6.5,7.0,7.5,8.0,8.5,9.0,9.5,10.0,10.5$, or $11.0\left(50 \mathrm{mM} \mathrm{KH}_{2} \mathrm{PO}_{4}-\mathrm{NaOH}\right.$ buffer for $\mathrm{pH} 6.5-8.0,50 \mathrm{mM}$ Tris- $\mathrm{HCl}$ buffer for $\mathrm{pH} 8.0-9.0,50 \mathrm{mM}$ glycine-NaOH buffer for $\mathrm{pH}$ 9.5-10.0, KCl-NaOH buffer for $\mathrm{pH}$ 11.0). Different concentrations of L-arginine $(5,10,20,30$, and $40 \mathrm{mM})$ were used to determine the optimal substrate concentration. All reactions were carried out in triplicate.

\subsection{Analytical Methods}

The concentrations of L-arginine, agmatine, and putrescine were determined by HPLC, as reported previously, with minor modifications [35]. The instrument was an Agilent 1260 Infinity (Agilent Technologies, Santa Clara, CA, USA), fitted with a Waters (Milford, MA, USA) Sunfire C18 column $(4.6 \times 250 \mathrm{~mm}, 5 \mu \mathrm{m})$, and an Agilent 1260 fluorescence detector G1321C. The column temperature was $25^{\circ} \mathrm{C}$, the flow rate was $1 \mathrm{~mL} \mathrm{~min}^{-1}$, and the injection volume was $10 \mu \mathrm{L}$. The mobile phases were: Solvent A-0.050 M acetate buffer/tetrahydrofuran (96/4, pH 6.0) and Solvent B-methanol. The percentage of Solvent B was $0 \%$ from 0 to $17 \mathrm{~min}$, ramped linearly to $33.3 \%$ at $22 \mathrm{~min}$, then to $100 \%$ at $30 \mathrm{~min}$, and maintained at $100 \%$ until $35 \mathrm{~min}$. The excitation and emission wavelengths were $340 \mathrm{~nm}$ and $420 \mathrm{~nm}$, respectively. The O-phthaldialdehyde reagents were from Agilent Technologies.

Author Contributions: Conceptualization, H.H. and Y.B.; methodology, T.-P.F.; software, H.H.; validation, Y.C. and T.-P.F.; formal analysis, H.H.; investigation, H.H.; resources, X.Z.; data curation, Y.C.; writing-original draft preparation, H.H.; writing-review and editing, H.H. and Y.C.; visualization, T.-P.F.; supervision, X.Z.; project administration, X.Z.; funding acquisition, Y.C. All authors have read and agreed to the published version of the manuscript.

Funding: We thank the National Key Scientific Instrument and Equipment Development Project of China (2013YQ17052504), the Program for Changjiang Scholars and Innovative Research Teams in the University of Ministry of Education of China (IRT_15R55), the Natural Science Foundation of Shanxi province (2019JQ-725), and the Postgraduate Research \& Practice Innovation Program of Jiangsu Province (KYCX19_1841, KYCX19_1842) for financial support.

Conflicts of Interest: The authors declare no conflict of interest.

\section{References}

1. Sugiyama, Y.; Nakamura, A.; Matsumoto, M.; Kanbe, A.; Sakanaka, M.; Higashi, K.; Igarashi, K.; Katayama, T.; Suzuki, H.; Kurihara, S. A novel putrescine exporter sapBCDF of Escherichia coli. J. Biol. Chem. 2016, 291, 26343-26351. [CrossRef] [PubMed]

2. Wallace, H.M.; Fraser, A.V.; Hughes, A. A perspective of polyamine metabolism. Biochem. J. 2003, 376, 1-14. [CrossRef] [PubMed]

3. Pegg, A.E.; McCann, P.P. Polyamine metabolism and function. Am. J. Physiol.-Cell Physiol. 1982, 243, C212-C221. [CrossRef] [PubMed]

4. Liu, G.; Zheng, J.; Wu, X.; Xu, X.; Jia, G.; Zhao, H.; Chen, X.; Wu, C.; Tian, G.; Wang, J. Putrescine enhances intestinal immune function and regulates intestinal bacteria in weaning piglets. Food Funct. 2019, 10, 4134-4142. [CrossRef]

5. Sugiyama, Y.; Nara, M.; Sakanaka, M.; Gotoh, A.; Kitakata, A.; Okuda, S.; Kurihara, S. Comprehensive analysis of polyamine transport and biosynthesis in the dominant human gut bacteria: Potential presence of novel polyamine metabolism and transport genes. Int. J. Biochem. Cell Biol. 2017, 93, 52-61. [CrossRef]

6. Chen, D.D.; Shao, Q.S.; Yin, L.H.; Younis, A.; Zheng, B.S. Polyamine function in plants: Metabolism, regulation on development, and roles in abiotic stress responses. Front. Plant. Sci. 2019, 9, 13. [CrossRef]

7. Yuan, Y.H.; Zhong, M.; Du, N.S.; Shu, S.; Sun, J.; Guo, S.R. Putrescine enhances salt tolerance of cucumber seedlings by regulating ion homeostasis. Environ. Exp. Bot. 2019, 165, 70-82. [CrossRef] 
8. Zheng, Q.L.; Zuo, J.H.; Gu, S.T.; Gao, L.P.; Hu, W.Z.; Wang, Q.; Jiang, A.L. Putrescine treatment reduces yellowing during senescence of broccoli (Brassica oleracea L. var. italica). Postharvest Biol. Technol. 2019, 152, 29-35. [CrossRef]

9. Scott, E.; Peter, F.; Sanders, J. Biomass in the manufacture of industrial products-The use of proteins and amino acids. Appl. Microbiol. Biotechnol. 2007, 75, 751-762. [CrossRef]

10. Qian, Z.G.; Xia, X.X.; Lee, S.Y. Metabolic engineering of Escherichia coli for the production of putrescine: A four carbon diamine. Biotechnol. Bioeng. 2009, 104, 651-662. [CrossRef]

11. Yamanobe, T.; Kurihara, Y.; Uehara, H.; Komoto, T. Structure and characterization of nylon 46. J. Mol. Struct. 2007, 829, 80-87. [CrossRef]

12. Choi, H.; Kyeong, H.-H.; Choi, J.M.; Kim, H.-S. Rational design of ornithine decarboxylase with high catalytic activity for the production of putrescine. Appl. Microbiol. Biotechnol. 2014, 98, 7483-7490. [CrossRef] [PubMed]

13. Schneider, J.; Wendisch, V.F.J.A.M. Putrescine production by engineered Corynebacterium glutamicum. Appl. Microbiol. Biotechnol. 2010, 88, 859-868. [CrossRef] [PubMed]

14. Schneider, J.; Eberhardt, D.; Wendisch, V.F. Improving putrescine production by Corynebacterium glutamicum by fine-tuning ornithine transcarbamoylase activity using a plasmid addiction system. Appl. Microbiol. Biotechnol. 2012, 95, 169-178. [CrossRef] [PubMed]

15. Nguyen, A.Q.D.; Schneider, J.; Reddy, G.K.; Wendisch, V.F. Fermentative production of the diamine putrescine: System metabolic engineering of Corynebacterium glutamicum. Metabolites 2015, 5, 211-231. [CrossRef]

16. Nguyen, A.Q.D.; Schneider, J.; Wendisch, V.F. Elimination of polyamine N-acetylation and regulatory engineering improved putrescine production by Corynebacterium glutamicum. J. Biotechnol. 2015, 201, 75-85. [CrossRef]

17. Meiswinkel, T.M.; Gopinath, V.; Lindner, S.N.; Nampoothiri, K.M.; Wendisch, V.F. Accelerated pentose utilization by Corynebacterium glutamicum for accelerated production of lysine, glutamate, ornithine and putrescine. Microb. Biotechnol. 2013, 6, 131-140. [CrossRef]

18. Del Rio, B.; Linares, D.M.; Ladero, V.; Redruello, B.; Fernandez, M.; Martin, M.C.; Alvarez, M.A. Putrescine production via the agmatine deiminase pathway increases the growth of Lactococcus lactis and causes the alkalinization of the culture medium. Appl. Microbiol. Biotechnol. 2015, 99, 897-905. [CrossRef]

19. Srinivasan, P.; Smolke, C.D. Engineering a microbial biosynthesis platform for de novo production of tropane alkaloids. Nat. Commun. 2019, 10, 15. [CrossRef]

20. France, S.P.; Hepworth, L.J.; Turner, N.J.; Flitsch, S.L. Constructing biocatalytic cascades: In vitro and in vivo approaches to de novo multi-enzyme pathways. ACS Catal. 2017, 7, 710-724. [CrossRef]

21. Li, C.; Jia, P.; Bai, Y.; Fan, T.P.; Zheng, X.; Cai, Y. Efficient synthesis of hydroxytyrosol from 1-3,4-dihydroxyphenylalanine using engineered Escherichia coli whole cells. J. Agric. Food Chem. 2019, 67, 6867-6873. [CrossRef] [PubMed]

22. Xiong, T.; Jiang, J.; Bai, Y.; Fan, T.-p.; Zhao, Y.; Zheng, X.; Cai, Y. Biosynthesis of D-danshensu from L-DOPA using engineered Escherichia coli whole cells. Appl. Microbiol. Biotechnol. 2019, 103, 6097-6105. [CrossRef] [PubMed]

23. Song, J.; Zhou, C.; Liu, R.; Wu, X.; Wu, D.; Hu, X.; Ding, Y. Expression and purification of recombinant arginine decarboxylase (speA) from Escherichia coli. Mol. Biol. Rep. 2010, 37, 1823-1829. [CrossRef] [PubMed]

24. Satishchandran, C.; Boyle, S.M. Purification and properties of agmatine ureohydrolyase, a putrescine biosynthetic enzyme in Escherichia coli. J. Bacteriol. 1986, 165, 843-848. [CrossRef] [PubMed]

25. Zhu, D.; Wu, J.; Zhan, X.; Zhu, L.; Zheng, Z.; Gao, M. Phosphoenolpyruvate-supply module in Escherichia coli improves N-acetyl-d-neuraminic acid biocatalysis. Biotechnol. Lett. 2017, 39, 227-234. [CrossRef]

26. Bentley, W.E.; Mirjalili, N.; Andersen, D.C.; Davis, R.H.; Kompala, D.S. Plasmid-encoded protein: The principal factor in the "metabolic burden" associated with recombinant bacteria. Biotechnol. Bioeng. 2009, 102, 1284-1297. [CrossRef]

27. Jones, K.L.; Kim, S.-W.; Keasling, J.D. Low-copy plasmids can perform as well as or better than high-copy plasmids for metabolic engineering of bacteria. Metab. Eng. 2000, 2, 328-338. [CrossRef]

28. Morris, D.R.; Koffron, K.L. Putrescine biosynthesis in Escherichia coli. regulation through pathway selection. J. Biol. Chem. 1969, 244, 6094-6099.

29. Wu, J.; Du, G.; Zhou, J.; Chen, J. Metabolic engineering of Escherichia coli for (2S)-pinocembrin production from glucose by a modular metabolic strategy. Metab. Eng. 2013, 16, 48-55. [CrossRef] 
30. Hou, Y.; Hossain, G.S.; Li, J.; Shin, H.D.; Du, G.; Chen, J.; Liu, L. Metabolic engineering of cofactor flavin adenine dinucleotide (FAD) synthesis and regeneration in Escherichia coli for production of alpha-keto acids. Biotechnol. Bioeng. 2017, 114, 1928-1936. [CrossRef]

31. Wu, W.H.; Morris, D.R. Biosynthetic arginine decarboxylase from Escherichia coli. purification and properties. J. Biol. Chem. 1973, 248, 1687-1695. [PubMed]

32. Forouhar, F.; Lew, S.; Seetharaman, J.; Xiao, R.; Acton, T.B.; Montelione, G.T.; Tong, L.A. Structures of bacterial biosynthetic arginine decarboxylases. Acta Crystallogr. F-Struct. Biol. Cryst. Commun. 2010, 66, 1562-1566. [CrossRef] [PubMed]

33. Ginesy, M.; Belotserkovsky, J.; Enman, J.; Isaksson, L.; Rova, U. Metabolic engineering of Escherichia coli for enhanced arginine biosynthesis. Microb. Cell. Fact. 2015, 14, 29. [CrossRef] [PubMed]

34. Najmi, Z.; Ebrahimipour, G.; Franzetti, A.; Banat, I.M. In situ downstream strategies for cost-effective bio/surfactant recovery. Biotechnol. Appl. Biochem. 2018, 65, 523-532. [CrossRef]

35. Yildirim, H.K.; Uren, A.; Yucel, U. Evaluation of biogenic amines in organic and non-organic wines by HPLC OPA derivatization. Food Technol. Biotechnol. 2007, 45, 62-68.

(C) 2020 by the authors. Licensee MDPI, Basel, Switzerland. This article is an open access article distributed under the terms and conditions of the Creative Commons Attribution (CC BY) license (http://creativecommons.org/licenses/by/4.0/). 